\title{
Energy-Momentum Density of Non-Diagonal Bianchi Type Space-Time in General Relativity and Teleparallel Gravity
}

\author{
Ragab M. Gad ${ }^{1,2}$, Haifaa A. Alharbi ${ }^{1}$ \\ ${ }^{1}$ Mathematics Department, Faculty of Science, University of Jeddah, North Jeddah, KSA \\ ${ }^{2}$ Mathematics Department, Faculty of Science, Minia University, El-Minia, Egypt \\ Email: ragab2gad@hotmail.com, haifaa.alharbi135@gmail.com
}

How to cite this paper: Gad, R.M. and Alharbi, H.A. (2021) Energy-Momentum Density of Non-Diagonal Bianchi Type Space-Time in General Relativity and Teleparallel Gravity. Journal of Applied Mathematics and Physics, 9, 2355-2375. https://doi.org/10.4236/jamp.2021.99150

Received: August 23, 2021

Accepted: September 25, 2021

Published: September 28, 2021

Copyright (c) 2021 by author(s) and Scientific Research Publishing Inc. This work is licensed under the Creative Commons Attribution International License (CC BY 4.0).

http://creativecommons.org/licenses/by/4.0/

\begin{abstract}
We calculated the energy-momentum density of non-diagonal Bianchi type space-time in two different theories of gravity, General relativity (GR) and the theory of Teleparallel gravity (TG). Firstly, by applying Einstein, LandauLifshitz, Bergmann-Thomson and Møller prescriptions, using double index complexes in GR. Secondly, in the frame work of TG, we used the energy momentum complexes of Einstein, Bergmann-Thomson and Landau-Lifshitz. We also study the spacial cases of non-diagonal Bianchi type space-time BII, BVIII and BIX. We obtained the same energy-momentum density components for Einstein and Bergmann-Thomson prescriptions for the above four mentioned space-times that we considered in our work. Also, we found that the energy density component in Møller prescription is zero for all Bianchi types space-times in GR. Furthermore, we show that if the metric components are functions of time $t$ alone, then the total gravitational energy is identically zero.
\end{abstract}

\section{Keywords}

Energy-Momentum Densities, Double Index Complexes, Teleparallel Gravity, Bianchi Type Space Time

\section{Introduction}

Since the outset of the general theory of relativity, the notion of energy-momentum localization has been one of the thorniest problems, which remains unsolved. There have been many attempts to find a generally accepted definition of the energy-momentum distributions for the gravitational field. Furthermore, there is no general agreed definition of energy in GR till now. Many tools have been 
found to manage the energy-momentum localization problem, such as the super-energy tensors [1] [2], and quasi-local definitions which are introduced by Penrose [3], and the most powerful tool of all the previously mentioned ones is the famous energy-momentum complexes which we are going to give a brief intro about it now. Einstein [4] himself believed that the energy can be localized in GR and he introduced to the world the first ever prescription to the energymomentum. Following Einstein attempt, several physicists proposed different energy-momentum complexes definitions, such as Tolman [5], Landau and Lifshitz [6], Papapetrou [7], Bergmann and Thomson [8], Weinberg [9] and Møller [10]. For a given space-time, these complexes give the same energy and momentum density see ([11] [12] [13] [14]). However, some obtained results showed that these complexes do not provide same results for example, see ([15]-[24]).

The main issue that makes energy-momentum complexes have some difficulties, was because using different energy-momentum prescriptions could lead to different energy distributions for same space-time, and all calculations should be done only in Cartesian coordinate expect for Møller. Besides that, the biggest lack was there is no precise definition of a pseudo tensor in the general relativity.

Some interesting results have been found recently by using different energy-momentum complexes in the general theory of relativity (see for example, [25] [26] [27] [28] [29]).

The problem of energy-momentum localization has reformulated to an alternative theory called Teleparallel Gravity, which concentrates on the gauge theories for the translation group based on Weitzenböck geometry. In the theory of the teleparallel gravity, gravitation is ascribed to torsion. This theory was attempted to solve the problems that are caused by the theory of general relativity. The first who noticed that the tetrad description of the gravitational field allows a more satisfactory treatment of the gravitational energy-momentum than it does in GR was Møller [30]. Within this theory, several researchers have calculated the energy-momentum distribution. For instance, Vargas [31], by using Einstein and Landau-Lifshitz energy-momentum complexes of the teleparallel version, found that total energy of the Friedmann-Robertson Walker space-time vanishes everywhere, which is agreed with the previous work of Cooperstock [32] and Rosen [33] in the concept of GR. Recently Alofi and Gad [34] obtained the TG versions of Lewis-Papapetrou Spacetime, and Aktacs [35] investigated Energy momentum distributions of Ruban universe in GR and TG. Gad and Alharbi [36] obtained the energy-momentum density for the gravitational field of the Van Stockum space-time in GR and TG.

Spatially, homogeneous cosmological models play a major role in understanding the structure and properties of space, and have been studied by many scientists. Rosen [33] and Cooperstock [32] used the Einstein complex to calculate the energy and momentum distributions of a closed homogeneous isotropic universe that is described by Friedmann Robertson Walker (FRW) space-time. They showed that the total energy of the Universe is equal to zero everywhere. 
Johri et al. [37] and Cooperstock and Israelit [38] calculated the total energy of of FRW Universe and they found it is also equal to zero at all times. Banerjee and Sen [39] studied the Bianchi type $I$ and $I I$ space-times by using the Einstein energy-momentum complex and they found that the total energy density is zero everywhere. Xulu [40] extended the investigations of Banerjee and Sen with three more energy-momentum complexes using Landau and Lifshitz, Papapetrou, and Weinberg and he found the same results. Radinschi [13] completed the investigation of Banerjee and Sen and Xulu for Bianchi type $I$ by using the energy-momentum complex of Tolman and her result agrees with the results of Banerjee and Sen and Xulu that the total energy density vanishes everywhere. Vargas and So [41] by working in the context of teleparallel gravity they calculated the quasilocal energy-momentum of the Bianchi type $I$ and $I I$ universes and they found that energy of both the matter and the gravitational fields vanishes for all regions this result is consistent with of Banerjee and Sen, Xulu, and Radinschi result that calculated in the framework of general relativity. Vargas [31] calculated the total energy of the FRW universe in teleparallel version of both Einstein and Landau-Lifshitz energy-momentum complexes and he found that the total energy vanishes whatever. Aydogdu and Salti [42] calculated the energy-momentum density for Bianchi type- $I I$ space time with the Bergmann-Thomson energy momentum definitions in both general relativity and teleparallel gravity and they found that these two gravitational theories give the same result which is that total energy is equal to zero. Tripathy et al. [43] they obtained energy and momentum distributions for the spatially homogeneous and anisotropic Bianchi type $\mathrm{VI}_{h}$ metric, they used Einstein, Landau-Lifshitz, Papapetrou, and Bergmann-Thompson complexes in the framework of general relativity. They found that the results only agree for a specific value of the metric parameter $h$, where the energy and momentum vanish for the Bianchi $\mathrm{VI}_{-1}$ universe, except for the case of Landau-Lifshitz where the energy density components vanish, the momentum density components do not vanish. Özkurt and Aygün [44] investigated the energy and momentum density homogeneous Bianchi type $\mathrm{VI}_{h}$ metric with Einstein, Bergmann-Thomson, Landau-Lifshitz, Papapetrou, Tolman and Møller prescriptions in general relativity and teleparallel gravity and their results agree with Tripathyet and Tryon.

Following by Mishra et al. [45] obtained the energy and momentum for diagonal Bianchi types they used Einstein, Landau-Lifshitz, Papapetrou, Bergmann-Thompson, and Møller energy-momentum complexes in GR and they found for $B I$ Universe the energy and momentum vanish identically for all prescriptions. Moreover for BIII, BV, $\mathrm{BVI}_{h}$ Universes they found that with specific choices of the metric parameter $h$ the energy of the Universes becomes zero, except for The Møller the energy density is found to be zero for all the Universes they have been considered. Recently Yang [27] investigated the energy of Bianchi type- $I I$ cosmological model by using two prescriptions the energy-momentum complexes of Einstein and Møller, and obtained that total energy is zero in both prescriptions. 
In the present work we illustrate the problem of energy and momentum density of non-diagonal Bianchi type space-time within the framework of two different theories of gravity, general relativity and teleparallel gravity. The paper has been organized as follows: In the next section we will briefly provide a brief overview of the non-diagonal Bianchi type space-time. In Section 3, we calculate the energy-momentum density for the above space-time in GR using double index complexes of Einstein, Møller, Landau-Lifshitz and Bergmann-Thomson. In section 4 we give a brief summary of the basic concepts of teleparallel gravity beside the calculation of the energy and momentum density components in the context of TG. In Section 5, we applied our obtained results to find the energy and momentum density of the non-diagonal Bianchi type BII BVIII and BIX Universe in both theories by using the same method. Finally, in section 6 we provide a summary and discussion of the obtained results.

Through this paper we will use $G=1$ and $c=1$ units and the Greek alphabet $(\mu, v, \rho=0,1,2,3)$ to denote tensor indices, that is indices related to space-time. The Latin alphabet $(a, b, c=0,1,2,3)$ will be used to denote local Lorentz (or tangent space) indices whose associated metric tensor is $\eta_{a b}=\operatorname{diag}(1,-1,-1,-1)$.

\section{Non-Diagonal Bianchi Type Space-Time}

The homogeneous and anisotropic Cosmological models have an important role in describing the universe in the early stages of its development [46]. The Bianchi cosmologies have a significant role in theoretical cosmology, these models was studied by several authors, see [40] [42] [43] [44] [45] [47] [48] [49] [50] [51]. There are nine Bianchi type divided into two special classes: class $A$ (Types I, II, $\mathrm{VI}_{0}, \mathrm{VII}_{0}$, VIII, IX), and class $B$ (type III, IV, V, $\mathrm{VI}_{\mathrm{h}}, \mathrm{VII}_{\mathrm{h}}$ ).

Now we consider the line element in terms of quasi-Cartesian coordinates:

$$
\begin{aligned}
\mathrm{d} s^{2}= & \mathrm{d} t^{2}-a_{1}^{2}(t) \mathrm{d} x_{1}^{2}-\left[\mathfrak{h}^{2}\left(x_{3}\right) a_{1}^{2}(t)+f^{2}\left(x_{3}\right) a_{2}^{2}(t)\right] \mathrm{d} x_{2}^{2} \\
& -a_{3}^{2} \mathrm{~d} x_{3}^{2}+2 a_{1}^{2}(t) \mathfrak{h}\left(x_{3}\right) \mathrm{d} x_{1} \mathrm{~d} x_{2}
\end{aligned}
$$

where $a_{1}(t), a_{2}(t), a_{3}(t)$ are functions of time and $f\left(x_{3}\right)$ and $\mathfrak{h}\left(x_{3}\right)$ are some functions of $x_{3}$. We use the following (see [51] [52]),

$$
\delta=-\frac{f^{\prime \prime}}{f},
$$

where prime denotes a partial derivative with respect to $x_{3}$,

$$
'=\frac{\partial}{\partial x_{3}} \text {. }
$$

According to the value of $\delta$ we classified the Bianchi type-II (BII), Bianchi type-VIII (BVIII) and Bianchi type-IX (BIX) models from the metric (2.1), respectively as follows:

$$
\begin{array}{ll}
\delta=0, & \text { corresponds to BII model, } \\
\delta=-1, & \text { corresponds to BVIII model, } \\
\delta=1, & \text { corresponds to BIX model. }
\end{array}
$$




\section{Energy-Momentum Complexes in General Relativity}

In the theory of General Relativity, the energy-momentum prescriptions of Einstein (E), Møller (M), Bergmann-Thomson (BT) and Landau-Lifshitz (LL) can be written as follows:

$$
\gamma_{i}^{k}=\partial_{\ell} U_{i}^{[k \ell]}, \quad \gamma^{i k}=\partial_{\ell} U^{i[k \ell]},
$$

with

$$
U_{i}^{[k \ell]}=\left\{\begin{array}{l}
\frac{1}{2 \kappa}(-g)^{-\frac{1}{2}} g_{i n} \partial_{m}\left[(-g)\left(g^{k n} g^{\ell m}-g^{\ell n} g^{k m}\right)\right] \\
\frac{1}{\kappa}(-g)^{\frac{1}{2}} g^{k m} g^{\ell n}\left(\partial_{m} g_{i n}-\partial_{n} g_{i m}\right)
\end{array}\right.
$$

where $\kappa=8 \pi$.

$$
U^{i[k \ell]}= \begin{cases}\frac{1}{2 \kappa}(-g)^{-\frac{1}{2}} \partial_{m}\left[(-g)\left(g^{i k} g^{\ell m}-g^{i \ell} g^{k m}\right)\right] & \text { (BT), } \\ \frac{1}{2 \kappa} \partial_{m}\left[(-g)\left(g^{i k} g^{\ell m}-g^{i \ell} g^{k m}\right)\right] & \text { (LL). }\end{cases}
$$

These complexes are called double index complexes [53].

The superpotential $U_{i}^{[k \ell]}$ or $U^{i[k \ell]}$ is made up from the contravariant components of the metric tensor and their derivative. By definition, each of them is anti-symmetric in the pair of indices $k$ and $\ell$, hence the complexes satisfy the local conservation law

$$
\partial_{k} \gamma_{i}^{k}=0, \quad \partial_{k} \gamma^{i k}=0
$$

$\gamma_{0}^{0}, \gamma^{00}$, and $\gamma_{\mu}^{0}, \gamma^{\mu 0}$ are, respectively, the energy and momentum density components. The complexes deriving from superpotential (3.3), are symmetrical in the two indices $i$ and $k$, this permits the establishment of a conservation law of angular momentum as well.

The energy and momentum distributions in the above various complexes are defined by

$$
\begin{aligned}
& P_{i}=\iiint \gamma_{i}^{0} \mathrm{~d}^{3} x=\int_{S} \int U_{i}^{[0 \ell]} \mathrm{d} S_{\ell}, \\
& P^{i}=\iiint \gamma^{i 0} \mathrm{~d}^{3} x=\int_{S} \int U^{i[0 \ell]} \mathrm{d} S_{\ell},
\end{aligned}
$$

where the first integration in both two above equations being carried out on the surface $x^{0}=$ const. and the second on a close two-surface $S$ belonging to the same hypersurface and expanding to infinity.

Now, if we assume the general metric

$$
\mathrm{d} s^{2}=g_{\mu \nu} \mathrm{d} x^{\mu} \mathrm{d} x^{v}
$$

where the metric components $g_{\mu v}$ are functions of time $t$ alone and free of space variables. Consequently, the superpotential $U_{i}^{[k \ell]}$ and $U^{i[k \ell]}$ are also functions of the time variable alone. From (3.1) and the antisymmetric of superpotential, we have

$$
\gamma_{0}^{0}=0, \quad \gamma^{00}=0
$$


Therefore,

Theorem 1. For all homogeneous space-times anzatz with the metric (3.6) the total gravitational energy for any finite volume is identically zero.

\subsection{Einstein Prescription}

The non-vanishing superpotential components, $U_{i}^{[k \epsilon]}$, of the Einstein complex for the line element (2.1), using Equation (3.2), are

$$
\begin{aligned}
& { }^{E} U_{0}^{[03]}=\frac{a_{1} a_{2} f^{\prime}}{\kappa a_{3}}, \\
& { }^{E} U_{1}^{[01]}=-\frac{1}{\kappa} a_{1} f\left(a_{2} \dot{a}_{3}+a_{3} \dot{a}_{2}\right), \\
& { }^{E} U_{1}^{[02]}=\frac{1}{\kappa} a_{3} \mathfrak{h} f\left(a_{1} \dot{a}_{2}-a_{2} \dot{a}_{1}\right), \\
& { }^{E} U_{2}^{[01]}=\frac{1}{\kappa} a_{3} \mathfrak{h} f\left(a_{1} \dot{a}_{2}-a_{2} \dot{a}_{1}\right), \\
& { }^{E} U_{2}^{[02]}=-\frac{1}{\kappa} a_{2} f\left(a_{1} \dot{a}_{3}+a_{3} \dot{a}_{1}\right), \\
& { }^{E} U_{3}^{[03]}=-\frac{1}{\kappa} a_{3} f\left(\dot{a}_{1} a_{2}+\dot{a}_{2} a_{1}\right) .
\end{aligned}
$$

where dot denotes a partial derivative with respect to $t$.

Inserting the above components into (3.1), we find the energy and momentum density components in the sense of Einstein definition, respectively, as follows

$$
\begin{aligned}
& { }^{E} \gamma_{0}^{0}=-\frac{1}{\kappa}\left[\frac{a_{1} a_{2} f^{\prime \prime}}{a_{3}}\right], \\
& { }^{E} \gamma_{3}^{0}=-\frac{1}{\kappa}\left[a_{3}\left(a_{1} \dot{a}_{2}+a_{2} \dot{a}_{1}\right) f^{\prime}\right], \\
& { }^{E} \gamma_{1}^{0}={ }^{E} \gamma_{2}^{0}=0 .
\end{aligned}
$$

\subsection{Møller Prescription}

The non-vanishing superpotential components, $U_{i}^{[k]]}$, of the Møller definition for the line element (2.1), using Equation (3.2), are

$$
\begin{aligned}
& { }^{M} U_{1}^{[01]}=\frac{2}{\kappa}\left(f a_{2} a_{3} \dot{a}_{1}\right), \\
& { }^{M} U_{2}^{[01]}=\frac{2}{\kappa}\left(a_{2} a_{3} f \mathfrak{h}\right)\left(a_{1} \dot{a}_{2}-a_{2} \dot{a}_{1}\right), \\
& { }^{M} U_{2}^{[02]}=\frac{2}{\kappa}\left(a_{1} a_{3} \dot{a}_{2} f\right), \\
& { }^{M} U_{3}^{[03]}=\frac{1}{\kappa}\left(2 a_{1} a_{2} \dot{a}_{3} f\right) .
\end{aligned}
$$

Inserting the above components into (1), we find the energy and momentum density components, respectively, as follows

$$
\begin{aligned}
& { }^{M} \gamma_{3}^{0}=\frac{1}{\kappa}\left(2 a_{1} a_{2} \dot{a}_{3} f^{\prime}\right), \\
& { }^{M} \gamma_{0}^{0}={ }^{M} \gamma_{1}^{0}={ }^{M} \gamma_{2}^{0}=0 .
\end{aligned}
$$




\subsection{Landau-Lifshitz Prescription}

The non-vanishing superpotential components, $U^{i[k \ell]}$, of the Landau-Lifshitz prescription for the line element (2.1), using Equation (3.3), are

$$
\begin{aligned}
& { }^{L L} U^{[003]}=-\frac{1}{\kappa}\left(a_{1}^{2} a_{2}^{2} f f^{\prime}\right), \\
& { }^{L L} U^{[101]}=\frac{1}{\kappa}\left[\left(a_{1} \dot{a}_{3}+\dot{a}_{1} a_{3}\right) a_{1} a_{3} \mathfrak{h}^{2}+\left(a_{2} \dot{a}_{3}+\dot{a}_{2} a_{3}\right) a_{2} a_{3} f^{2}\right], \\
& { }^{L L} U^{[102]}={ }^{B T} U^{[201]}=\frac{1}{\kappa}\left(\dot{a}_{3} a_{1}+\dot{a}_{1} a_{3}\right) a_{1} a_{3} \mathfrak{h}, \\
& { }^{L L} U^{[202]}=\frac{1}{\kappa}\left(\dot{a}_{3} a_{1}+\dot{a}_{1} a_{3}\right) a_{1} a_{3}, \\
& { }^{L L} U^{[303]}=\frac{1}{\kappa}\left(\dot{a}_{1} a_{2}+\dot{a}_{2} a_{1}\right) a_{1} a_{2} f^{2} .
\end{aligned}
$$

Using the above components in (3.1), we obtain the Landau-Lifshitz's energy and momentum density components, respectively, as follows

$$
\begin{aligned}
{ }^{L L} \gamma^{00} & =-\frac{1}{\kappa} a_{1}^{2} a_{2}^{2}\left(f f^{\prime \prime}+\left(f^{\prime}\right)^{2}\right), \\
{ }^{L L} \gamma^{30} & =\frac{2}{\kappa}\left(\dot{a}_{1} a_{2}+\dot{a}_{2} a_{1}\right) a_{1} a_{2} f f^{\prime}, \\
{ }^{L L} \gamma^{10} & ={ }^{L L} \gamma^{20}=0 .
\end{aligned}
$$

\subsection{Bergmann-Thomson Prescription}

The non-vanishing superpotential components, $U^{i[k \ell]}$, of the Bergmann-Thomson complex for the line element (2.1), using Equation (3.3), are

$$
\begin{aligned}
& { }^{B T} U^{[003]}=\frac{1}{\kappa}\left[\frac{a_{1} a_{2} f^{\prime}}{a_{3}}\right], \\
& { }^{B T} U^{[101]}=\frac{1}{\kappa} \frac{1}{a_{1} a_{2} f}\left[a_{2} f^{2}\left(\dot{a}_{3} a_{2}+a_{2} \dot{a}_{3}\right)+a_{1} \mathfrak{h}^{2}\left(\dot{a}_{3} a_{1}+a_{3} \dot{a}_{1}\right)\right], \\
& { }^{B T} U^{[102]}={ }^{B T} U^{[201]}=\frac{\mathfrak{h}}{\kappa} \frac{1}{a_{2} f}\left(\dot{a}_{1} a_{3}+\dot{a}_{3} a_{1}\right), \\
& { }^{B T} U^{[202]}=\frac{1}{\kappa} \frac{1}{a_{2} f}\left(\dot{a}_{1} a_{3}+\dot{a}_{3} a_{1}\right), \\
& { }^{B T} U^{[303]}=\frac{1}{\kappa} \frac{1}{a_{3}}\left(\dot{a}_{1} a_{2}+\dot{a}_{2} a_{1}\right) f .
\end{aligned}
$$

Inserting the above components into (3.1), we get the energy and momentum density components using Bergmann-Thomson's prescription, respectively, as follows

$$
\begin{aligned}
& { }^{B T} \gamma^{00}=-\frac{1}{\kappa}\left[\frac{a_{1} a_{2} f^{\prime \prime}}{a_{3}}\right], \\
& { }^{B T} \gamma^{30}=\frac{1}{\kappa} \frac{1}{a_{3}}\left(\dot{a}_{1} a_{2}+\dot{a}_{2} a_{1}\right) f^{\prime}, \\
& { }^{B T} \gamma^{10}={ }^{B T} \gamma^{20}=0 .
\end{aligned}
$$




\section{Teleparallel Gravity}

The name of teleparallel gravity is usually used to denote the general threeparameter theory [54]. Teleparallel gravity theory has the same field equations as the theory of general relativity. It is an alternative formulation of gravity. In other words, it is equivalent to the theory of general relativity [55]. It can actually be understand as a gauge theory for the translation group based on Weitzenböck geometry [56].

\subsection{Fundamental Concepts}

In this section we are going to review the basic concepts of the theory of teleparallel gravity (see for example [54] [57] [58] [59]).

In the theory of teleparallel gravity the tetrad, $h_{\mu}^{a}$, is play the main role, while the metric tensor play the main role in the theory of general relativity. There are many ways to create tetrad, $h_{\mu}^{a}$, one of these ways, it can be expressed by the dual basis of the differential one-form by choosing a coframe of the coordinate system [60]. Another way is by solving the following relation,

$$
g_{\mu v}=\eta_{a b} h_{\mu}^{a} h_{v}^{b}
$$

where $\eta_{a b}=\operatorname{diag}(1,-1,-1,-1)$.

$h_{\mu}^{a}$ whose inverse is denoted by, $h_{a}^{v}$, should satisfy the relationships

$$
h_{\mu}^{a} h_{a}^{v}=\delta_{\mu}^{v} ; \quad h_{\mu}^{a} h_{b}^{\mu}=\delta_{b}^{a},
$$

and

$$
h=\operatorname{det}\left(h_{\mu}^{a}\right)=\sqrt{-g}, \text { where } g:=\operatorname{det}\left(g_{\mu \nu}\right),
$$

where $\left(g_{\mu v}\right)$ is the covarint metric.

Using the Minkowski metric, $\eta_{a b}$, the tangent space indices are raised and lowered while by using the space-time metric, $g_{\mu v}$, the space-time indices are raised and lowered. The parallel transport of $h_{v}^{a}$ between two neighboring points is coded into the covariant derivative

$$
\nabla_{\mu} h_{v}^{a}=\partial_{\mu} h_{v}^{a}-\Gamma_{\mu v}^{\alpha} h_{\alpha}^{a},
$$

where

$$
\Gamma_{\mu v}^{\alpha}=h_{a}^{\alpha} \partial_{v} h_{\mu}^{a}=-h_{\mu}^{a} \partial_{v} h_{a}^{\alpha}
$$

is a Weitzenböck connection [56]. This connection provides a torsion that is defined as follows

$$
T_{\mu v}^{\rho}=\Gamma_{v \mu}^{\rho}-\Gamma_{\mu v}^{\rho}=h_{a}^{\alpha}\left(\partial_{\mu} h_{v}^{a}-\partial_{v} h_{\mu}^{a}\right)
$$

It is not symmetric in $\mu$ and $v$.

The connection $\Gamma_{\mu v}^{\rho}$ and the Levi-Civita connection $\tilde{\Gamma}_{\mu v}^{\rho}{ }^{1}$ are connected by the following relation

$$
\Gamma_{\mu v}^{\rho}=\tilde{\Gamma}_{\mu v}^{\rho}+K_{\mu v}^{\rho},
$$

${ }^{1} \tilde{\Gamma}_{\mu \nu}^{\rho}=\frac{1}{2} g^{\rho \sigma}\left(g_{\mu \sigma, \nu}+g_{v \sigma, \mu}-g_{\mu v, \sigma}\right)$. 
where

$$
K_{\mu \nu}^{\rho}=\frac{1}{2}\left(T_{\mu \nu}^{\rho}+T_{\nu \mu}^{\rho}-T_{\mu \nu}^{\rho}\right)
$$

is called the contortion tensor.

The Weitzenböck connection $T_{\lambda \mu v}$ can be defined by the following three parts [54]

$$
T_{\lambda \mu \nu}=\frac{1}{2}\left(t_{\lambda \mu \nu}-t_{\lambda v \mu}\right)+\frac{1}{3}\left(g_{\lambda \mu} V_{v}-g_{\lambda v} V_{\mu}\right)+\varepsilon_{\lambda \mu v \rho} A^{\rho}
$$

where

$$
t_{\lambda \mu \nu}=\frac{1}{2}\left(T_{\lambda \mu v}+T_{\mu \lambda v}\right)+\frac{1}{6}\left(g_{\nu \lambda} V_{\mu}+g_{\mu \nu} V_{\lambda}\right)-\frac{1}{3} g_{\lambda \mu} V_{v}
$$

is the tensor part that represents the torsion tensor,

$$
V_{\mu}=T_{v \mu}^{v}
$$

is the vector part that gives the torsion vector, and

$$
A^{\mu}=h_{a}^{\mu} A^{a}=\frac{1}{6} \varepsilon^{\mu \nu \rho \sigma} T_{\nu \rho \sigma}
$$

is the axial-vector part that defines the torsion axial-vector, which represents the axial symmetry deviation from spherical symmetry [61].

$\varepsilon^{\mu v \rho \sigma}$ and $\varepsilon_{\mu \nu \rho \sigma}$ are completely antisymmetric tensors with respect to the coordinates basis and defined by [62]

$$
\begin{gathered}
\varepsilon^{\mu \nu \rho \sigma}=\frac{1}{\sqrt{-g}} \delta^{\mu \nu \rho \sigma}, \\
\varepsilon_{\mu v \rho \sigma}=\sqrt{-g} \delta_{\mu \nu \rho \sigma},
\end{gathered}
$$

where $\delta^{\mu v \rho \sigma}$ and $\delta_{\mu \nu \rho \sigma}$ are the antisymmetric tensor densities of weight -1 and +1 , respectively, with normalization $\delta_{0123}=-1$ and $\delta^{0123}=+1$.

In the presence of matter, the action of teleparallel gravity is given by

$$
\mathcal{A}=\frac{1}{16 \pi} \int \mathrm{d}^{4} x h S^{\rho \mu v} T_{\rho \mu v}+\int \mathrm{d}^{4} x h £_{M},
$$

where $h=\operatorname{det}\left(h_{\mu}^{a}\right), f_{M}$ is the Lagrangian of a source field and

$$
S^{\rho \mu v}=c_{1} T^{\rho \mu v}+\frac{c_{2}}{2}\left(T^{\mu \rho v}-T^{v \rho \mu}\right)+\frac{c_{3}}{2}\left(g^{\rho v} T_{\sigma}^{\sigma \mu}-g^{\mu \rho} T_{\sigma}^{\sigma v}\right) .
$$

is a tensor written in terms of the torsion of the Weitzenböck connection. In the above form, $c_{1}, c_{2}$ and $c_{3}$ are the three dimensionless coupling constants of Teleparallel Gravity.

These constants has a defined value that has been determined by Hayashi [54]

$$
c_{1}=\frac{1}{4}, \quad c_{2}=\frac{1}{2}, \quad c_{3}=-1 .
$$

The energy-momentum complexes of Einstein, Bergmann-Thomson and Landau-Lifshitz in Teleparallel Gravity, respectively, are given by [30] 


$$
\begin{aligned}
h E^{\mu}{ }_{v} & =\frac{1}{4 \pi} \partial_{\lambda}\left(\mho_{v}^{\mu \lambda}\right), \\
h B^{\mu \nu} & =\frac{1}{4 \pi} \partial_{\lambda}\left(g^{\mu \beta} \mho_{\beta}^{v \lambda}\right), \\
h L^{\mu v} & =\frac{1}{4 \pi} \partial_{\lambda}\left(h g^{\mu \beta} \mho_{\beta}^{v \lambda}\right),
\end{aligned}
$$

where $\mho_{v}^{\mu \lambda}$ is the Freud's super-potential and defined as follows

$$
\mho_{v}^{\mu \lambda}=h S_{v}^{\mu \lambda} .
$$

The energy and momentum distributions in the above complexes, respectively, are

$$
\begin{aligned}
& { }^{E} P_{\mu}=\int_{\Sigma} h E_{\mu}^{0} \mathrm{~d}^{3} x, \\
& { }^{B T} P_{\mu}=\int_{\Sigma} h B_{\mu}^{0} \mathrm{~d}^{3} x, \\
& { }^{L L} P_{\mu}=\int_{\Sigma} h L_{\mu}^{0} \mathrm{~d}^{3} x,
\end{aligned}
$$

where $P_{0}$ is the energy, $P_{i}(i=1,2,3)$ are the momentum components and the integration hypersurface $\Sigma$ is described by $x^{0}=t$ constant.

\subsection{Energy-Momentum Density in Teleparallel Gravity}

In order to calculate the Einstein, Bergmann-Thomson and Landau-Lifshitz's energy and momentum complexes of the metric in teleparallel gravity, we consider the tetrad components of the line element (2.1). The tetrad components represented by $h_{\mu}^{a}$ as follows

$$
h_{\mu}^{a}=\left[\begin{array}{cccc}
1 & 0 & 0 & 0 \\
0 & a_{1} & -a_{1} \mathfrak{h} & 0 \\
0 & 0 & a_{2} f & 0 \\
0 & 0 & 0 & a_{3}
\end{array}\right],
$$

and its inverse $h_{a}^{\mu}=g^{\mu v} \eta_{a b} h_{v}^{b}$ is

$$
h_{a}^{\mu}=\left[\begin{array}{cccc}
1 & 0 & 0 & 0 \\
0 & \frac{1}{a_{1}} & \frac{\mathfrak{h}}{a_{2} f} & 0 \\
0 & 0 & \frac{1}{a_{2} f} & 0 \\
0 & 0 & 0 & \frac{1}{a_{3}}
\end{array}\right],
$$

We can inspect that (4.21) and (4.22) satisfy the tetrad conditions (4.2), and hence the condition

$$
h:=\operatorname{det} h_{\mu}^{a}=\sqrt{-g}, \text { where } g:=\operatorname{det} g_{\mu \nu},
$$

so

$$
h:=\operatorname{det} h_{\mu}^{a}=a_{1} a_{2} a_{3} f .
$$

Using the above components of $h_{\mu}^{a}$ and $h_{a}^{\mu}$ in (4.5) we construct the Weit- 
zenböck connection, whose non-vanishing components are

$$
\begin{aligned}
\Gamma_{10}^{1} & =\frac{\dot{a}_{1}}{a_{1}}, \\
\Gamma_{23}^{1} & =-\mathfrak{h}^{\prime}+\frac{\mathfrak{h} f^{\prime}}{f}, \\
\Gamma_{20}^{1} & =\frac{-\dot{a}_{1} \mathfrak{h}}{a_{1}}+\frac{\mathfrak{h} \dot{a}_{2}}{a_{2}}, \\
\Gamma_{20}^{2} & =\frac{\dot{a}_{2}}{a_{2}}, \\
\Gamma_{23}^{2} & =\frac{f^{\prime}}{f}, \\
\Gamma_{30}^{3} & =\frac{\dot{a}_{3}}{a_{3}} .
\end{aligned}
$$

Using (4.6) and the above components, we find the non-vanishing torsion components as following

$$
\begin{aligned}
& T_{10}^{1}=-T_{10}^{1}=-\Gamma_{10}^{1}, \\
& T_{20}^{1}=-T_{02}^{1}=-\Gamma_{20}^{1}, \\
& T_{23}^{1}=-T_{32}^{1}=-\Gamma_{23}^{1}, \\
& T_{20}^{2}=-T_{02}^{2}=-\Gamma_{20}^{2}, \\
& T_{23}^{2}=-T_{32}^{2}=-\Gamma_{23}^{2}, \\
& T_{30}^{3}=-T_{03}^{3}=-\Gamma_{30}^{3} .
\end{aligned}
$$

Using this torsion component into Equation (4.16), we found the non-vanishing components of the tensor $\mathcal{S}_{\beta}^{\mu v}$ as follows

$$
\begin{aligned}
S_{0}^{03} & =-\frac{f^{\prime}}{2 a_{3}^{2} f}, \\
S_{1}^{01} & =\frac{-\dot{a}_{3} a_{2}-\dot{a}_{2} a_{3}}{2 a_{2} a_{3}}, \\
S_{1}^{02} & =\frac{a_{1} \mathfrak{h}(\mathfrak{h}-1)\left(a_{1} \dot{a}_{2}-a_{2} \dot{a}_{1}\right)}{4 a_{2}^{3} f^{2}}, \\
S_{1}^{13} & =\frac{-a_{1}^{2} \mathfrak{h} \mathfrak{h}^{\prime}-2 f^{\prime} f a_{2}^{2}}{4 f^{2} a_{3}^{2} a_{2}^{2}}, \\
S_{1}^{23} & =\frac{a_{1}^{2} \mathfrak{h}^{\prime}}{4 a_{3}^{2} f^{2} a_{2}^{2}}, \\
S_{2}^{01} & =\frac{-\mathfrak{h}\left(a_{2} \dot{a}_{1}-a_{1} \dot{a}_{2}\right)}{2 a_{1} a_{2}}, \\
S_{2}^{02} & =\frac{-\dot{a}_{3} a_{1}-\dot{a}_{1} a_{3}}{2 a_{1} a_{3}}, \\
S_{2}^{13} & =\frac{\mathfrak{h}\left[\left(f^{2} a_{2}^{2}+a_{3}^{2} \mathfrak{h}\right) f^{\prime}+f \mathfrak{h}^{\prime}\left(a_{1}^{2} \mathfrak{h}-a_{3}^{2}\right)\right]}{4 a_{3}^{2} f^{3} a_{2}^{2}}, \\
S_{2}^{23} & =\frac{a_{1}^{2} \mathfrak{h} \mathfrak{h}^{\prime}}{4 a_{3}^{2} f^{2} a_{2}^{2}},
\end{aligned}
$$




$$
\begin{aligned}
& S_{3}^{03}=\frac{-\dot{a}_{1}}{2 a_{1}}-\frac{\dot{a}_{2}}{2 a_{2}}, \\
& S_{3}^{12}=\frac{\mathfrak{h}^{\prime}}{4 f^{2} a_{2}^{2}} .
\end{aligned}
$$

Using these components (4.25) in the relation (4.19) then insert it in equations (4.18), we obtain the energy and momentum densities in the sense of Einstein, Landau-Lifshitz, and Bergmann-Thomson, respectively, as follows.

The energy-momentum density components in the Einstein prescription will become

$$
\begin{aligned}
& h E_{0}^{0}=-\frac{1}{\kappa}\left[\frac{a_{1} a_{2} f^{\prime \prime}}{a_{3}}\right], \\
& h E_{0}^{3}=-\frac{1}{\kappa}\left[a_{3}\left(a_{1} \dot{a}_{2}+a_{2} \dot{a}_{1}\right) f^{\prime}\right], \\
& h E_{0}^{1}=h E_{0}^{2}=0 .
\end{aligned}
$$

The energy-momentum density components in the Landau-Lifshitz prescription will become

$$
\begin{aligned}
& h L^{00}=-\frac{1}{\kappa} a_{1}^{2} a_{2}^{2}\left(f f^{\prime \prime}+\left(f^{\prime}\right)^{2}\right), \\
& h L^{03}=\frac{2}{\kappa}\left(\dot{a}_{1} a_{2}+\dot{a}_{2} a_{1}\right) a_{1} a_{2} f f^{\prime}, \\
& h L^{01} h L^{02}=0 .
\end{aligned}
$$

The energy-momentum density components in the Bergmann-Thomson prescription will become

$$
\begin{aligned}
h B^{00} & =-\frac{1}{\kappa}\left[\frac{a_{1} a_{2} f^{\prime \prime}}{a_{3}}\right], \\
h B^{03} & =\frac{1}{\kappa} \frac{1}{a_{3}}\left(\dot{a}_{1} a_{2}+\dot{a}_{2} a_{1}\right) f^{\prime}, \\
h B^{01} & =h B^{02}=0 .
\end{aligned}
$$

\section{Special Cases}

According to the value of $\delta$ using (2.3), we define $f\left(x_{3}\right)$, the function $\mathfrak{h}\left(x_{3}\right)$ can be defined from the solution of the Einstein field equations as follows [51] [52].

$$
\mathfrak{h}^{\prime \prime}-\frac{f^{\prime}}{f} \mathfrak{h}^{\prime}=b f, \quad b=\text { const. }
$$

We consider from the general case of the Bianchi type space-time the following Special Cases.

Case I

The metric (2.1) reduces to $B I I$ space time if $\delta=0$

$$
-\frac{f^{\prime \prime}}{f}=0, \rightarrow f^{\prime \prime}=0 .
$$

Solving the above equation, we get 


$$
f\left(x_{3}\right)=c_{1} x_{3}+c_{2},
$$

where $c_{1}, c_{2}$ being some arbitrary constants. Without loss of generality, when $f=x_{3}$ and by solving the non-homogeneous linear differential Equation (5.1) we find

$$
\mathfrak{h}\left(x_{3}\right)=b x_{3}^{3} / 3+c_{3} x_{3}^{2} / 2+c_{4},
$$

where $c_{3}, c_{4}$ being some arbitrary constants.

Then the corresponding metric is expressed by the line element

$$
\begin{aligned}
\mathrm{d} s^{2}= & \mathrm{d} t^{2}-a_{1}^{2} d x_{1}^{2}-\left[\left(b^{2} x_{3}^{6} / 9+b x_{3}^{5} / 3+x_{3}^{4} / 4\right) a_{1}^{2}+x_{3}^{2} a_{2}^{2}\right] \mathrm{d} x_{2}^{2} \\
& -a_{3}^{2} \mathrm{~d} x_{3}^{2}+2\left[a_{1}^{2}\left(b x_{3}^{3} / 3+x_{3}^{2} / 2\right)\right] \mathrm{d} x_{1} \mathrm{~d} x_{2} .
\end{aligned}
$$

Assuming that $b=0$ then the line element becomes

$$
\mathrm{d} s^{2}=\mathrm{d} t^{2}-a_{1}^{2} \mathrm{~d} x_{1}^{2}-\left[\frac{1}{4} x_{3}^{4} a_{1}^{2}+x_{3}^{2} a_{2}^{2}\right] \mathrm{d} x_{2}^{2}-a_{3}^{2} \mathrm{~d} x_{3}^{2}+2\left[\frac{1}{2} a_{1}^{2} x_{3}^{2}\right] \mathrm{d} x_{1} \mathrm{~d} x_{2} .
$$

\section{Case II}

When $\delta=-1$, we get $B V I I I$ space-time

$$
-\frac{f^{\prime \prime}}{f}=-1, \quad \rightarrow f^{\prime \prime}-f=0 .
$$

This is a homogeneous equation has the general solution as

$$
f\left(x_{3}\right)=c_{5} \sinh \left(x_{3}\right)+c_{6} \cosh \left(x_{3}\right),
$$

where $c_{5}, c_{6}$ being some arbitrary constants. Without loss of generality, when $f\left(x_{3}\right)=\sinh \left(x_{3}\right)$ and by solving equation (5.1), we find

$$
\mathfrak{h}\left(x_{3}\right)=b x_{3} \cosh x_{3}-b \sinh \left(x_{3}\right)+c_{7} \cosh \left(x_{3}\right)+c_{8},
$$

where $c_{7}, c_{8}$ being some arbitrary constants.

Then the corresponding metric takes the form,

$$
\begin{aligned}
\mathrm{d} s^{2}= & \mathrm{d} t^{2}-a_{1}^{2} \mathrm{~d} x_{1}^{2}-\left[\left(b^{2} x^{2} \cosh ^{2}\left(x_{3}\right)-2 b^{2} x \sinh \left(x_{3}\right) \cosh \left(x_{3}\right)\right.\right. \\
& +2 b x \cosh ^{2}\left(x_{3}\right)+b^{2} \sinh ^{2}\left(x_{3}\right)-2 b \cosh \left(x_{3}\right) \sinh \left(x_{3}\right) \\
& \left.\left.+\cosh ^{2}\left(x_{3}\right)\right) a_{1}^{2}+\sinh ^{2}\left(x_{3}\right) a_{2}^{2}\right] \mathrm{d} x_{2}^{2}-a_{3}^{2} \mathrm{~d} x_{3}^{2} \\
& +2 a_{1}^{2}\left[b x_{3} \cosh \left(x_{3}\right)-b \sinh \left(x_{3}\right)+\cosh \left(x_{3}\right)\right] \mathrm{d} x_{1} \mathrm{~d} x_{2} .
\end{aligned}
$$

Assuming that $b=0$ then the line element become as follows,

$$
\begin{aligned}
\mathrm{d} s^{2}= & \mathrm{d} t^{2}-a_{1}^{2} \mathrm{~d} x_{1}^{2}-\left[\cosh ^{2}\left(x_{3}\right) a_{1}^{2}+\sinh ^{2}\left(x_{3}\right) a_{2}^{2}\right] \mathrm{d} x_{2}^{2} \\
& -a_{3}^{2} \mathrm{~d} x_{3}^{2}+2 a_{1}^{2} \cosh \left(x_{3}\right) \mathrm{d} x_{1} \mathrm{~d} x_{2} .
\end{aligned}
$$

\section{Case III}

Finally, the $B I X$ space-time can be found when $\delta=1$

$$
-\frac{f^{\prime \prime}}{f}=1, \quad \rightarrow f^{\prime \prime}+f=0 .
$$

Solving the above equation, we have the general solution as follows 


$$
f\left(x_{3}\right)=c_{9} \sin \left(x_{3}\right)+c_{10} \cos \left(x_{3}\right),
$$

with $c_{9}, c_{10}$ being some arbitrary constants. Without loss of generality, when $f\left(x_{3}\right)=\sin \left(x_{3}\right)$, the solution of (5.1) becomes

$$
\mathfrak{h}\left(x_{3}\right)=-\left(b x_{3}\right) \cos \left(x_{3}\right)+b \sin \left(x_{3}\right)-c_{11} \cos \left(x_{3}\right)+c_{12},
$$

where $c_{11}, c_{12}$ being some arbitrary constants.

Therefore, the corresponding metric takes the form,

$$
\begin{aligned}
\mathrm{d} s^{2}= & \mathrm{d} t^{2}-a_{1}^{2} \mathrm{~d} x_{1}^{2}-\left[\left(b^{2} x^{2} \cos \left(x_{3}\right)+2 b^{2} x \sin \left(x_{3}\right) \cos \left(x_{3}\right)+2 b x \cos ^{2}\left(x_{3}\right)\right.\right. \\
& \left.\left.+b^{2} \sin ^{2}\left(x_{3}\right)+2 b \cos \left(x_{3}\right) \sin \left(x_{3}\right)+\cos ^{2}(x)\right) a_{1}^{2}+\sin ^{2}\left(x_{3}\right) a_{2}^{2}\right] \mathrm{d} x_{2}^{2} \\
& -a_{3}^{2} \mathrm{~d} x_{3}^{2}+2 a_{1}^{2}\left[\left(-b x_{3}\right) \cos \left(x_{3}\right)+b \sin \left(x_{3}\right)-\cos \left(x_{3}\right)\right] \mathrm{d} x_{1} \mathrm{~d} x_{2} .
\end{aligned}
$$

Assuming that $b=0$ then the line element is expressed by,

$$
\begin{aligned}
\mathrm{d} s^{2}= & \mathrm{d} t^{2}-a_{1}^{2} \mathrm{~d} x_{1}^{2}-\left[\cos ^{2}\left(x_{3}\right) a_{1}^{2}+\sin ^{2}\left(x_{3}\right) a_{2}^{2}\right] \mathrm{d} x_{2}^{2} \\
& -a_{3}^{2} \mathrm{~d} x_{3}^{2}+2 a_{1}^{2} \cos \left(x_{3}\right) \mathrm{d} x_{1} \mathrm{~d} x_{2} .
\end{aligned}
$$

Now we are going to obtain the energy-momentum density in GR by using Einstein's, Møller, Landau-Lifshitz and Bergman-Thompson prescriptions, and then in TG by using Einstein's, Landau-Lifshitz and Bergman-Thompson energy-momentum complexes.

\section{Einstein prescription}

The energy-momentum density components in the sense of Einstein, respectively, as follows

For the $B I I$ space-time represented by the line element (5.4),

$$
\begin{aligned}
{ }^{E} \gamma_{0}^{0} & =0, \\
{ }^{E} \gamma_{3}^{0} & =-\frac{1}{\kappa}\left[a_{3}\left(a_{1} \dot{a}_{2}+a_{2} \dot{a}_{1}\right)\right], \\
{ }^{E} \gamma_{1}^{0} & ={ }^{E} \gamma_{2}^{0}=0 . \\
h E_{0}^{0} & =0, \\
h E_{0}^{3} & =-\frac{1}{\kappa}\left[a_{3}\left(a_{1} \dot{a}_{2}+a_{2} \dot{a}_{1}\right)\right], \\
h E_{0}^{1} & =h E_{0}^{2}=0 .
\end{aligned}
$$

and for the BVIII space-time showed by the line element (5.7),

$$
\begin{aligned}
{ }^{E} \gamma_{0}^{0} & =-\frac{1}{\kappa}\left[\frac{a_{1} a_{2} \sinh \left(x_{3}\right)}{a_{3}}\right], \\
{ }^{E} \gamma_{3}^{0} & =-\frac{1}{\kappa}\left[a_{3}\left(a_{1} \dot{a}_{2}+a_{2} \dot{a}_{1}\right) \cosh \left(x_{3}\right)\right], \\
{ }^{E} \gamma_{1}^{0} & ={ }^{E} \gamma_{2}^{0}=0 . \\
h E_{0}^{0} & =-\frac{1}{\kappa}\left[\frac{a_{1} a_{2} \sinh \left(x_{3}\right)}{a_{3}}\right], \\
h E_{0}^{3} & =-\frac{1}{\kappa}\left[a_{3}\left(a_{1} \dot{a}_{2}+a_{2} \dot{a}_{1}\right) \cosh \left(x_{3}\right)\right], \\
h E_{0}^{1} & =h E_{0}^{2}=0 .
\end{aligned}
$$


and for the $B I X$ space-time expressed by the line element (5.10),

$$
\begin{aligned}
& { }^{E} \gamma_{0}^{0}=\frac{1}{\kappa}\left[\frac{a_{1} a_{2} \sin \left(x_{3}\right)}{a_{3}}\right], \\
& { }^{E} \gamma_{3}^{0}=-\frac{1}{\kappa}\left[a_{3}\left(a_{1} \dot{a}_{2}+a_{2} \dot{a}_{1}\right) \cos \left(x_{3}\right)\right], \\
& { }^{E} \gamma_{1}^{0}={ }^{E} \gamma_{2}^{0}=0 . \\
& h E_{0}^{0}=\frac{1}{\kappa}\left[\frac{a_{1} a_{2} \sin \left(x_{3}\right)}{a_{3}}\right], \\
& h E_{0}^{3}=-\frac{1}{\kappa}\left[a_{3}\left(a_{1} \dot{a}_{2}+a_{2} \dot{a}_{1}\right) \cos \left(x_{3}\right)\right], \\
& h E_{0}^{1}=h E_{0}^{2}=0 .
\end{aligned}
$$

\section{Møller prescription}

The energy-momentum density components using the Møller prescription are as follows,

For the BII space-time represented by the line element (5.4),

$$
\begin{aligned}
& { }^{M} \gamma_{3}^{0}=\frac{2}{\kappa}\left(a_{1} a_{2} \dot{a}_{3}\right) \\
& { }^{M} \gamma_{0}^{0}={ }^{M} \gamma_{1}^{0}={ }^{M} \gamma_{2}^{0}=0 .
\end{aligned}
$$

and for the BVIII space-time defined by the line element (5.7),

$$
\begin{aligned}
& { }^{M} \gamma_{3}^{0}=\frac{1}{\kappa}\left(2 a_{1} a_{2} \dot{a}_{3} \cosh \left(x_{3}\right)\right), \\
& { }^{M} \gamma_{0}^{0}={ }^{M} \gamma_{1}^{0}={ }^{M} \gamma_{2}^{0}=0 .
\end{aligned}
$$

and for the $B I X$ space-time showed by the line element (5.10),

$$
\begin{aligned}
& { }^{M} \gamma_{3}^{0}=\frac{1}{\kappa}\left(2 a_{1} a_{2} \dot{a}_{3} \cos \left(x_{3}\right)\right), \\
& { }^{M} \gamma_{0}^{0}={ }^{M} \gamma_{1}^{0}={ }^{M} \gamma_{2}^{0}=0 .
\end{aligned}
$$

\section{Landau-Lifshitz prescription}

We obtain the Landau-Lifshitz's energy-momentum density components, respectively, as follows

For the BII space-time expressed by the line element (5.4),

$$
\begin{aligned}
{ }^{L L} \gamma^{00} & =-\frac{1}{\kappa} a_{1}^{2} a_{2}^{2}, \\
{ }^{L L} \gamma^{30} & =\frac{2}{\kappa}\left(\dot{a}_{1} a_{2}+\dot{a}_{2} a_{1}\right) a_{1} a_{2} x_{3}, \\
{ }^{L L} \gamma^{10} & ={ }^{L L} \gamma^{20}=0 . \\
h L^{00} & =-\frac{1}{\kappa} a_{1}^{2} a_{2}^{2}, \\
h L^{03} & =\frac{2}{\kappa}\left(\dot{a}_{1} a_{2}+\dot{a}_{2} a_{1}\right) a_{1} a_{2} x_{3}, \\
h L^{01} & =h L^{02}=0 .
\end{aligned}
$$

and for the $B V I I I$ space-time represented by the line element (5.7), 


$$
\begin{aligned}
{ }^{L L} \gamma^{00} & =-\frac{1}{\kappa} a_{1}^{2} a_{2}^{2}\left(\sinh ^{2}\left(x_{3}\right)+\cosh ^{2}\left(x_{3}\right)\right), \\
{ }^{L L} \gamma^{30} & =\frac{2}{\kappa}\left(\dot{a}_{1} a_{2}+\dot{a}_{2} a_{1}\right) a_{1} a_{2} \sinh \left(x_{3}\right) \cosh \left(x_{3}\right), \\
{ }^{L L} \gamma^{10} & ={ }^{L L} \gamma^{20}=0 . \\
h L^{00} & =-\frac{1}{\kappa} a_{1}^{2} a_{2}^{2}\left(\sinh ^{2}\left(x_{3}\right)+\cosh ^{2}\left(x_{3}\right)\right), \\
h L^{03} & =\frac{2}{\kappa}\left(\dot{a}_{1} a_{2}+\dot{a}_{2} a_{1}\right) a_{1} a_{2} \sinh \left(x_{3}\right) \cosh \left(x_{3}\right), \\
h L^{01} & =h L^{02}=0 .
\end{aligned}
$$

and for the $B I X$ space-time showed by the line element (5.10),

$$
\begin{aligned}
{ }^{L L} \gamma^{00} & =-\frac{1}{\kappa} a_{1}^{2} a_{2}^{2}\left(-\sin ^{2}\left(x_{3}\right)+\cos ^{2}\left(x_{3}\right)\right), \\
{ }^{L L} \gamma^{30} & =\frac{2}{\kappa}\left(\dot{a}_{1} a_{2}+\dot{a}_{2} a_{1}\right) a_{1} a_{2} \sin \left(x_{3}\right) \cos \left(x_{3}\right), \\
{ }^{L L} \gamma^{10} & ={ }^{L L} \gamma^{20}=0 . \\
h L^{00} & =-\frac{1}{\kappa} a_{1}^{2} a_{2}^{2}\left(-\sin ^{2}\left(x_{3}\right)+\cos ^{2}\left(x_{3}\right)\right), \\
h L^{03} & =\frac{2}{\kappa}\left(\dot{a}_{1} a_{2}+\dot{a}_{2} a_{1}\right) a_{1} a_{2} \sin \left(x_{3}\right) \cos \left(x_{3}\right), \\
h L^{01} & =h L^{02}=0 .
\end{aligned}
$$

\section{Bergmann-Thomson prescription}

We obtain the components of energy and momentum densities using the Bergmann-Thomson definition, respectively, as follows

For the $B I I$ space-time represented by the line element (5.4),

$$
\begin{aligned}
{ }^{B T} \gamma^{00} & =0, \\
{ }^{B T} \gamma^{30} & =\frac{1}{\kappa} \frac{1}{a_{3}}\left(\dot{a}_{1} a_{2}+\dot{a}_{2} a_{1}\right), \\
{ }^{B T} \gamma^{10} & ={ }^{B T} \gamma^{20}=0 . \\
h B^{00} & =0, \\
h B^{03} & =\frac{1}{\kappa} \frac{1}{a_{3}}\left(\dot{a}_{1} a_{2}+\dot{a}_{2} a_{1}\right), \\
h B^{01} & =h B^{02}=0 .
\end{aligned}
$$

For the $B V I I I$ space-time defined by the line element (5.7),

$$
\begin{aligned}
{ }^{B T} \gamma^{00} & =-\frac{1}{\kappa}\left[\frac{a_{1} a_{2} \sinh \left(x_{3}\right)}{a_{3}}\right], \\
{ }^{B T} \gamma^{30} & =\frac{1}{\kappa} \frac{1}{a_{3}}\left(\dot{a}_{1} a_{2}+\dot{a}_{2} a_{1}\right) \cosh \left(x_{3}\right), \\
{ }^{B T} \gamma^{10} & ={ }^{B T} \gamma^{20}=0 . \\
h B^{00} & =-\frac{1}{\kappa}\left[\frac{a_{1} a_{2} \sinh \left(x_{3}\right)}{a_{3}}\right], \\
h B^{03} & =\frac{1}{\kappa} \frac{1}{a_{3}}\left(\dot{a}_{1} a_{2}+\dot{a}_{2} a_{1}\right) \cosh \left(x_{3}\right),
\end{aligned}
$$




$$
h B^{01}=h B^{02}=0 .
$$

For the $B I X$ space-time represented by the line element (5.10),

$$
\begin{aligned}
{ }^{B T} \gamma^{00} & =\frac{1}{\kappa}\left[\frac{a_{1} a_{2} \sin \left(x_{3}\right)}{a_{3}}\right], \\
{ }^{B T} \gamma^{30} & =\frac{1}{\kappa} \frac{1}{a_{3}}\left(\dot{a}_{1} a_{2}+\dot{a}_{2} a_{1}\right) \cos \left(x_{3}\right), \\
{ }^{B T} \gamma^{10} & ={ }^{B T} \gamma^{20}=0 . \\
h B^{00} & =\frac{1}{\kappa}\left[\frac{a_{1} a_{2} \sin \left(x_{3}\right)}{a_{3}}\right], \\
h B^{03} & =\frac{1}{\kappa} \frac{1}{a_{3}}\left(\dot{a}_{1} a_{2}+\dot{a}_{2} a_{1}\right) \cos \left(x_{3}\right), \\
h B^{01} & =h B^{02}=0 .
\end{aligned}
$$

\section{Discussion and Summary}

In the present work, we have obtained the energy and momentum density components for the non diagonal Bianchi types for some well known energy-momentum complexes such as Einstein, Landau-Lifshitz, Bergmann-Thompson and Møller prescriptions using double index complexes in GR and as well as in TG. The general results of the non diagonal Bianchi type universes have been used to calculate the energy and momentum of BII, BVIII and BIX Universes.

Our result indicates the following:

1) We obtained that the Einstein prescription agrees with Bergmann-Thompson prescription. In these two prescriptions, we get the same energy-momentum density in GR as well as in TG,

$$
\begin{aligned}
& { }^{E} \gamma_{0}^{0}={ }^{B T} \gamma^{00} \\
& { }^{E} \gamma_{i}^{0}={ }^{B T} \gamma^{i 0}
\end{aligned}
$$

2) The only non vanishing momentum density components are the third momentum component. Otherwise, it vanishes identically for all prescriptions in both theories

$$
\begin{gathered}
{ }^{E} \gamma_{1}^{0}={ }^{E} \gamma_{2}^{0}={ }^{L L} \gamma^{10}={ }^{L L} \gamma^{20}={ }^{B T} \gamma^{10}={ }^{B T} \gamma^{20}={ }^{M} \gamma_{1}^{0}={ }^{M} \gamma_{2}^{0}=0 . \\
h E_{0}^{1}=h E_{0}^{2}=h B^{01}=h B^{02}=h L^{01}=h L^{02}=0 .
\end{gathered}
$$

3) Many researchers investigated the Bianchi type-II cosmological model in both theories and they indicated that the energy vanishes for all regions, see [27] [41] [50] [63] [64]. Our result agrees with them in the case of Einstein, Bergmann-Thompson, and Møller.

4) It is clear that the energy density in the Møller prescription is zero for all the non-diagonal Bianchi types in GR. Our result agrees with [45] where they obtained the energy-momentum density of some diagonal anisotropic Bianchi type Universes using different energy-momentum complexes in the framework of General Relativity. They found that unlike the other prescriptions, in the case 
of Møller, the energy is zero for all types of Bianchi Universes they have considered. Also, our result agrees with [49]. They found that Møller is the only prescription where the energy density is equal to zero for Bianchi type-IV Universe. Lassner [65] indicates that Møller energy-momentum complex is a powerful concept of energy and momentum in general relativity. Our result agrees with him.

5) While, in other prescriptions the energy density does not vanish for all types expect for Bianchi type-II space-time as we already indicated.

6) If the scale factors of the metric are function in $t$ only, then the total energy-momentum density is identically zero for all Bianchi types space-times. This sustains our theorem (1).

7) In all prescriptions, the expressions for the energy and momentum density are well-defined.

\section{Conflicts of Interest}

The authors declare no conflicts of interest regarding the publication of this paper.

\section{References}

[1] Bonilla, M.Á. and Senovilla, J.M. (1997) Some Properties of the Bel and Bel-Robinson Tensors. General Relativity and Gravitation, 29, 91-116. https://doi.org/10.1023/A:1010256231517

[2] Senovilla, J.M. (2000) Super-Energy Tensors. Classical and Quantum Gravity, 17, 2799. https://doi.org/10.1088/0264-9381/17/14/313

[3] Penrose, R. (1982) Quasi-Local Mass and Angular Momentum in General Relativity. Proceedings of the Royal Society of London. A. Mathematical and Physical Sciences, 381, 53-63. https://doi.org/10.1098/rspa.1982.0058

[4] Einstein, A. (1915) On the General Theory of Relativity. Sitzungsber. Preuss. Akad. Wiss. Berlin (Math. Phys.), 1915, 778-786.

[5] Tolman, R.C. (1934) Relativity, Thermodynamics, and Cosmology. Oxford University Press, London, 227.

[6] Landau, L.D. and Lifshitz, E.M. (2002) The Classical Theory of Fields. 4th Edition, Pergamon Press, Oxford.

[7] Papapetrou, A. (1948) Einstein's Theory of Gravitation and Flat Space. Proceedings of the Royal Irish Academy. Section A: Mathematical and Physical Sciences, 52 $11-23$.

[8] Bergmann, P.G. and Thomson, R. (1953) Spin and Angular Momentum in General Relativity. Physical Review, 89, 400. https://doi.org/10.1103/PhysRev.89.400

[9] Weinberg, S. (1972) Gravitation and Cosmology: Principles and Applications of the General Theory of Relativity. John Wiley \& Sons, Inc., Hoboken.

[10] Møller, C. (1958) On the Localization of the Energy of a Physical System in the General Theory of Relativity. Annals of Physics, 4, 347-371. https://doi.org/10.1016/0003-4916(58)90053-8

[11] Virbhadra, K. (1990) Energy Associated with a Kerr-Newman Black Hole. Physical Review D, 41, 1086. https://doi.org/10.1103/PhysRevD.41.1086 
[12] Vagenas, E.C. (2006) Energy Distribution in the Dyadosphere of a Reissner-Nordström Black Hole in Møller's Prescription. Modern Physics Letters A, 21, 1947-1956. https://doi.org/10.1142/S0217732306020482

[13] Radinschi, I. (2000) Energy of a Conformal Scalar Dyon Black Hole. Modern Physics Letters A, 15, 2171-2175. https://doi.org/10.1142/S0217732300002498

[14] Radinschi, I. and Grammenos, T. (2006) Energy Distribution of the Einstein-KleinGordon System for a Static Spherically Symmetric Space-Time in $(2+1)$ Dimensions. International Journal of Modern Physics A, 21, 2853-2861. https://doi.org/10.1142/S0217751X06031776

[15] Gad, R.M. (2004) Energy Distribution of a Gödel-Type Space-Time. Astrophysics and Space Science, 293, 453-460.

https://doi.org/10.1023/B:ASTR.0000044624.38810.da

[16] Gad, R.M. (2004) Energy and Momentum Associated with a Static Axially Symmetric Vacuum Spacetime. Modern Physics Letters A, 19, 1847-1854. https://doi.org/10.1142/S0217732304014744

[17] Gad R.M. (2013) On Teleparallel Version of Stationary Axisymmetric Solutions and Their Energy Contents. Astrophysics and Space Science, 346, 553-557. https://link.springer.com/article/10.1007/s10509-013-1476-4

[18] Abdel-Megied, M. and Gad, R.M. (2010) Møller's Energy in the Kantowski-Sachs Space-Time. Advances in High Energy Physics, 2010, Article ID: 379473.

https://www.hindawi.com/journals/ahep/2010/379473/

[19] Gad, R.M. (2006) Møller Energy-Momentum Complex of a Static Axially Symmetric Vacuum Space-Time. Astrophysics and Space Science, 302, 141-144. https://doi.org/10.1007/s10509-005-9014-7

[20] Gad, R.M. and Fouad, A. (2007) Energy and Momentum Distributions of Kantowski and Sachs Space-Time. Astrophysics and Space Science, 310, 135-140. https://doi.org/10.1007/s10509-007-9488-6

[21] Gad, R.M. and Mourad, M. (2008) Energy Distributions of Kantowski-Sachs SpaceTime in the Theory of Teleparallel Gravity. Astrophysics and Space Science, 314, 341-346. https://doi.org/10.1007/s10509-008-9775-x

[22] Gad, R.M. (2005) Energy Distribution of a Stringy Charged Black Hole. Astrophysics and Space Science, 295, 459-462. https://doi.org/10.1007/s10509-005-1195-6

[23] Gad, R.M. (2006) Energy and Momentum Associated with Solutions Exhibiting Directional Type Singularities. General Relativity and Gravitation, 38, 417-424. https://doi.org/10.1007/s10714-006-0230-4

[24] Gad, R.M. (2006) On the Energy-Momentum Distribution in Weyl Metrics. Nuovo Cimento B Serie, 121, 161-166.

[25] Ahmed, F. (2018) The Energy-Momentum Distributions and Relativistic Quantum Effects on Scalar and Spin-Half Particles in a Gödel-Type Space-Time. The European Physical Journal C, 78, Article No. 598. https://doi.org/10.1140/epjc/s10052-018-6082-8

[26] Sahoo, P., Radinschi, I. and Mahanta, K. (2020) Energy-Momentum Distribution in General Relativity for a Phantom Black Hole Metric. Indian Journal of Physics, 94, 2065-2072. https://doi.org/10.1007/s12648-019-01651-Z

[27] Yang, I.-C. (2019) The Energy of the Universe in the Bianchi Type-II Cosmological Model. Modern Physics Letters A, 34, Article ID: 1950192. https://doi.org/10.1142/S021773231950192X

[28] Radinschi, I., Grammenos, T., Rahaman, F., Cazacu, M.-M., Spanou, A. and Cha- 
kraborty, J. (2020) On the Energy of a Non-Singular Black Hole Solution Satisfying the Weak Energy Condition. Universe, 6, 169.

https://doi.org/10.3390/universe6100169

[29] Radinschi, I., Grammenos, T., Sahoo, P.K., Chattopadhyay, S. and Cazacu, M.M. (2021) Einstein and Møller Energies of a Particular Asymptotically Reissner-Nordström Non-Singular Black Hole Solution. Astronomische Nachrichten, 342, 264-270. https://doi.org/10.1002/asna.202113917

[30] Møller, C. (1962) Tetrad Fields and Conservation Laws in General Relativity. Academic Press, London.

[31] Vargas, T. (2004) The Energy of the Universe in Teleparallel Gravity. General Relativity and Gravitation, 36, 1255-1264. https://doi.org/10.1023/B:GERG.0000022386.29438.be

[32] Cooperstock, F. (1994) Perspectives on the Energy of the Universe. General Relativity and Gravitation, 26, 323-327. https://doi.org/10.1007/BF02108014

[33] Rosen, N. (1994) The Energy of the Universe. General Relativity and Gravitation, 26, 319-321. https://doi.org/10.1007/BF02108013

[34] Alofi, A. and Gad, R.M. (2019) On Torsion Axial Vector and Gravitational Energy in Lewis-Papapetrou Space-Time in the Theory of Teleparallel Gravity. arXiv preprint arXiv:1912.06653.

[35] Aktaş, C. (2019) Energy-Momentum Distributions of Ruban Universe in General Relativity and Teleparallel Gravity. International Journal of Modern Physics A, 34, Article ID: 1950011. https://doi.org/10.1142/S0217751X19500118

[36] Gad, R.M. and Alharbi, H.A. (2021) Gravitational Energy in Van Stockum Space-Time. Indian Journal of Physics, 1-7. https://doi.org/10.1007/s12648-021-02085-2

[37] Johri, V., Kalligas, D., Singh, G. and Everitt, C. (1995) Gravitational Energy in the Expanding Universe. General Relativity and Gravitation, 27, 313-318. https://doi.org/10.1007/BF02109127

[38] Cooperstock, F. and Israelit, M. (1995) The Energy of the Universe. Foundations of Physics, 25, 631-635. https://doi.org/10.1007/BF02059009

[39] Banerjee, N. and Sen, S. (1997) Einstein Pseudotensor and Total Energy of the Universe. Pramana, 49, 609-615. https://doi.org/10.1007/BF02848334

[40] Xulu, S.S. (2000) Total Energy of the Bianchi Type I Universes. International Journal of Theoretical Physics, 39, 1153-1161. https://doi.org/10.1023/A:1003670928681

[41] So, L.L. and Vargas, T. (2005) The Energy of Bianchi Type I and II Universes in Teleparallel Gravity. Chinese Journal of Physics, 43, 901-908.

[42] Aydoğdu, O. and Salti, M. (2005) Energy of the Universe in Bianchi-Type I Models in Møller's Tetrad Theory of Gravity. Astrophysics and Space Science, 299, 227-232. https://doi.org/10.1007/s10509-005-7216-7

[43] Tripathy, S., Mishra, B., Pandey, G., Singh, A., Kumar, T. and Xulu, S. (2015) Energy and Momentum of Bianchi Type $\mathrm{VI}_{h}$ Universes. Advances in High Energy Physics, 2015, Article ID: 705262. https://doi.org/10.1155/2015/705262

[44] Özkurt, S. and Aygün, S. (2017) Energy Distributions of Bianchi Type-VI ${ }_{h}$ Universe in General Relativity and Teleparallel Gravity. Pramana, 88, Article No. 66. https://doi.org/10.1007/s12043-017-1367-2

[45] Mishra, P.K., Panda, B., Pattanayak, P.R. and Tripathy, S.K. (2016) Tryon's Conjecture and Energy and Momentum of Bianchi Type Universes. Advances in High Energy Physics, 2016, Article ID: 1986387. https://doi.org/10.1155/2016/1986387 
[46] Bali, R. and Jain, D.R. (1989) An Anisotropic Magnetized Viscous Fluid Cosmological Model in General Relativity. International Journal of Theoretical Physics, 28, 903-910. https://doi.org/10.1007/BF00670003

[47] Radinschi, I. (2001) Energy Associated with the Bianchi Type VI0 Universe. Chinese Journal of Physics, 39, 393-396.

[48] Radinschi, I. (2000) The Energy Distribution of the Bianchi Type I Universe. arXiv preprint grqc/0008034.

[49] Aygün, S. and Tarhan, I. (2012) Energy-Momentum Localization for Bianchi Type-IV Universe in General Relativity and Teleparallel Gravity. Pramana, 78, 531-548. https://doi.org/10.1007/s12043-012-0261-1

[50] Nester, J.M., So, L.L. and Vargas, T. (2008) Energy of Homogeneous Cosmologies. Physical Review D, 78, Article ID: 044035. https://doi.org/10.1103/PhysRevD.78.044035

[51] Saha, B. (2013) Some Remarks on Bianchi Type-II, VIII, and IX Models. Gravitation and Cosmology, 19, 65-69. https://doi.org/10.1134/S0202289313010088

[52] Saha, B. (2018) Nonlinear Spinor Field in Non-Diagonal Bianchi Type Space-Time. EPJ Web of Conferences, 173, Article ID: 02018. https://doi.org/10.1051/epjconf/201817302018

[53] Cattaneo, C. (1966) Conservation Laws. Annales de P.H.P. Physique Théorique, 4, $1-20$.

[54] Hayashi, K. and Shirafuji, T. (1979) New General Relativity. Physical Review D, 19, 3524. https://doi.org/10.1103/PhysRevD.19.3524

[55] Hammond, R.T. (2002) Torsion Gravity. Reports on Progress in Physics, 65, 599. https://doi.org/10.1088/0034-4885/65/5/201

[56] Weitzenbock, R. (1923) Invariantentheorie. P. Noordhoff, Grønningen.

[57] Aldrovandi, R. and Pereira, J.G. (1995) An Introduction to Geometrical Physics. World Scientific, Singapore. https://doi.org/10.1142/2722

[58] De Andrade, V. and Pereira, J. (1997) Gravitational Lorentz Force and the Description of the Gravitational Interaction. Physical Review D, 56, 4689.

https://doi.org/10.1103/PhysRevD.56.4689

[59] De Andrade, V., Guillen, L. and Pereira, J. (2000) Gravitational Energy-Momentum Density in Teleparallel Gravity. Physical Review Letters, 84, 4533.

https://doi.org/10.1103/PhysRevLett.84.4533

[60] Hehl, F.W. and Ni, W.-T. (1990) Inertial Effects of a Dirac Particle. Physical Review $D, 42,2045$. https://doi.org/10.1103/PhysRevD.42.2045

[61] Nitsch, J. and Hehl, F.W. (1980) Translational Gauge Theory of Gravity: PostNewtonian Approximation and Spin Precession. Physics Letters B, 90, 98-102. https://doi.org/10.1016/0370-2693(80)90059-3

[62] Møller, C. (1972) The Theory of Relativity. Oxford University Press, Oxford.

[63] Aydogdu, O. (2006) Gravitational Energy-Momentum Density in Bianchi Type II Space-Times. International Journal of Modern Physics D, 15, 459-468. https://doi.org/10.1142/S0218271806008255

[64] Aydogdu, O. (2006) Energy Distribution of the Universe in the Bianchi Type II Cosmological Models. Fortschritte der Physik: Progress of Physics, 54, 246-251. https://doi.org/10.1002/prop.200510271

[65] Lessner, G. (1996) Møller's Energy-Momentum Complex-Once Again. General Relativity and Gravitation, 28, 527-535. https://doi.org/10.1007/BF02105063 